\title{
Mandibular Actinomyces Infection Mimicking a Malignancy: Case Report
}

\author{
Gülay BULUT'1 , Yasemin BAYRAM² ${ }^{2}$, Mehmet Deniz BULUT³ ${ }^{3}$, Mehmet Fatih GARÇA $^{4}$, İrfan BAYRAM ${ }^{1}$
}

Departments of ${ }^{1}$ Pathology, ${ }^{2}$ Microbiology, ${ }^{3}$ Radiology, and ${ }^{4}$ Otorhinolaryngology, Yüzüncü Yıl University, Faculty of Medicine, VAN, TURKEY

\begin{abstract}
Actinomycosis is a rare, chronic, suppurative and granulomatous disease caused by Actinomyces israelii, which is a filamentous, anaerobic, gram-positive, saprophytic organism in the oral cavity. Diagnosis of actinomycosis depends on positive culture or identification of Actinomyces colonies and sulfur granules in histological specimens. In our case, a mass had been growing in the mandible for eight months. The mass appeared to be malignant, both clinically and radiologically. A histopathological examination of the mandible revealed actinomycosis. It should be noted that actinomycosis can mimic a malignancy, and for differential diagnosis, bone biopsy or fine-needle aspiration should be performed pre-operatively.
\end{abstract}

Key Words: Cervicofacial actinomycosis, Pathology, Mandible

\section{INTRODUCTION}

The microorganisms classified as Actinomyces are common inhabitants of the oral cavity and the human pharynx region. Actinomycotic infection in chronic inflammation has specific effects on primary soft tissues, rarely affecting the bones (1). After the destruction of the oral mucosa, the anaerobic, filamentous, gram-positive, saprophytic and slow-growing bacterium Actinomyces israelii develops (2). It widely affects the neck, mandible, tonsils, hard palate, paranasal sinus, lacrimal gland, parotid gland, and the orbit in the cervicofacial area $(3,4)$. Alveolar bone and mandibular corpus actinomycosis is rare. The pathogenic mechanism underlying actinomycotic infiltration is unknown (5).

The first incidence of actinomycosis in humans was defined as a fungal infection by Von Langenbeck in 1845 . In 1891, Israel and Ponfick described the anaerobic nature of Actinomyces. In 1960, Waksman demonstrated that Actinomyces were gram-positive bacteria (5).

The cervical region, and particularly the submandibular area, is the main region that is affected in actinomycosis. Diagnosis is based on fine-needle aspiration cytology and biopsy. Computed tomography and magnetic resonance imaging can be useful for determining the size of the lesion. It is known that this disease has a tendency to resemble carcinoma and tuberculosis (5).

(Turk Patoloji Derg 2017, 33:256-258)

Received : 08.12.2013 Accepted : 09.05.2014

\section{CASE REPORT}

The case was a 16-year-old male patient who described a growing, painless mass in the left cheek for eight months. There was no history of trauma, although scar formation was noted on the skin. With deep palpation, there was no pain or heat associated with the palpation. A lack of teeth was observed during the oral cavity examination. Hematological examination revealed that the white blood cell count was at the high end of the normal range $\left(11.1 \times 10^{9} / \mathrm{L}\right.$; normal range, $4-11 \times 10^{9} / \mathrm{L}$ ). Tomography revealed significant cortical irregularity at the level of the left mandibular corpus condyle and the mandibular angulus as well as at the medullary area. An expansile mass of approximately $63 \times 23 \times 45 \mathrm{~mm}$ in size causing sclerosis with the formation of new bone around the bone, including multilocular cystic spaces, was observed. An evaluation of the tomography findings indicated ameloblastoma (Figure 1A,B). The patient was then referred to our center. The patient was operated on according to the results of tomography. No positive cultures were obtained from the wound site after the operation. The materials removed for macroscopic examination were a tissue sample $10 \times 2.5 \times 2.5 \mathrm{~cm}$ in size with a hard consistency compatible with mandibular bone and a mass $6.5 \times 3 \times 2.5 \mathrm{~cm}$ in size with two teeth. Severe active chronic inflammation, abscess formation, and osteomyelitis were observed on

Correspondence: Gülay BULUT

Yüzüncü Yıl Üniversitesi Tip Fakültesi, Patoloji Anabilim Dalı,

VAN, TURKEY

E-mail: drgulaybulut@yahoo.com Phone: +90 4322150473 
histopathological examination (Figure 2). Sulfur granules were observed via histochemical staining, and filamentous bacilli that stained positive with Gomori's methenamine silver stain (GMS) (Figure 3) and Periodic acid-Schiff stain (PAS) were also observed. The patient was diagnosed with an Actinomyces infection. After 2 years, the patient remained well and did not show signs of infectious relapse.
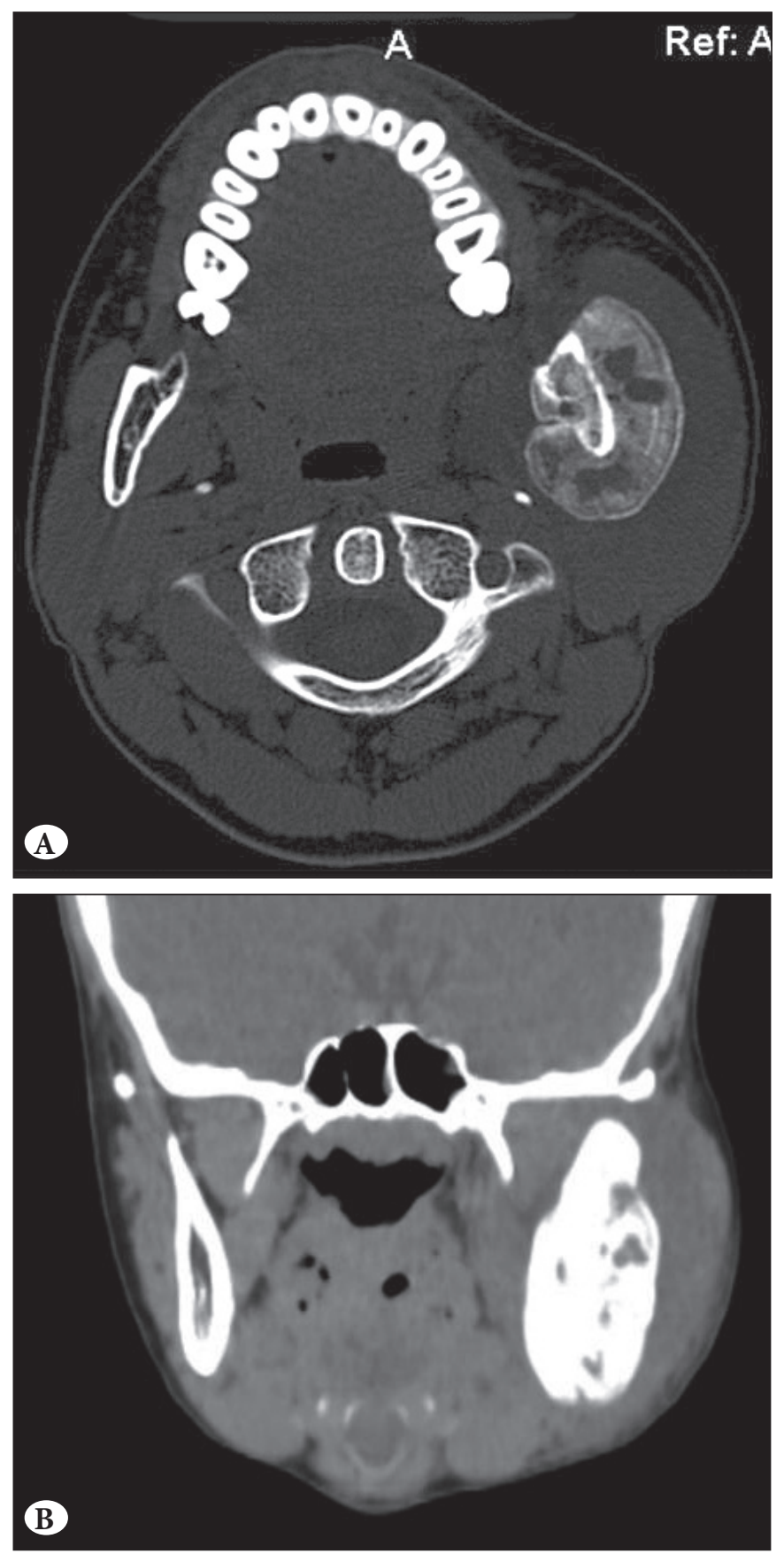

Figure 1: Axial bone window CT (A) and coronal CT (B) scans demonstrate an expansive, lytic-sclerotic bone lesion with welldefined margins affecting the left angle/ramus of the mandible.

\section{DISCUSSION}

Primary actinomycotic osteomyelitis is rare, corresponding to approximately $12 \%$ of osteomyelitis cases (6). It affects the cervicofacial region, typically the body of the mandible, followed by the region of the chin, branch, and angle of the mandible; however, it rarely affects the upper jaw or temporomandibular joint. Its prevalence in the mandible in relation to the maxilla is $4: 1$, as reported in the present case (1).

Actinomycosis infections usually involve the cervicofacial, thoracic, and abdominopelvic regions as well as the cranionervous system, and no person-to-person transmission has been documented. Actinomycotic osteomyelitis mainly occurs due to adjacent tissue infection, but it can also be observed in some fractures or in hematogenous spread. Cutaneous sinus tracts usually develop (6).

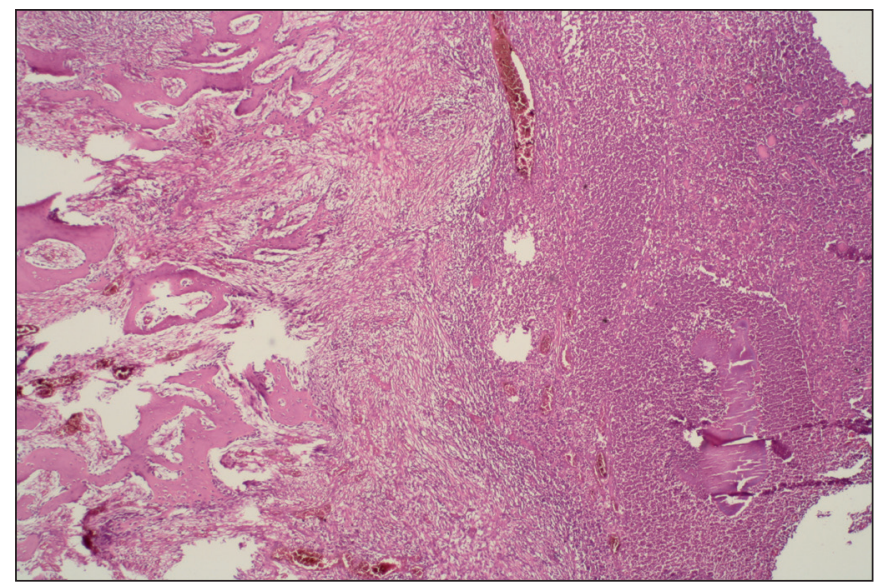

Figure 2: Identification of osteomyelitis and the location of the abscess in the mandible (HE, $\mathrm{x} 40$ ).

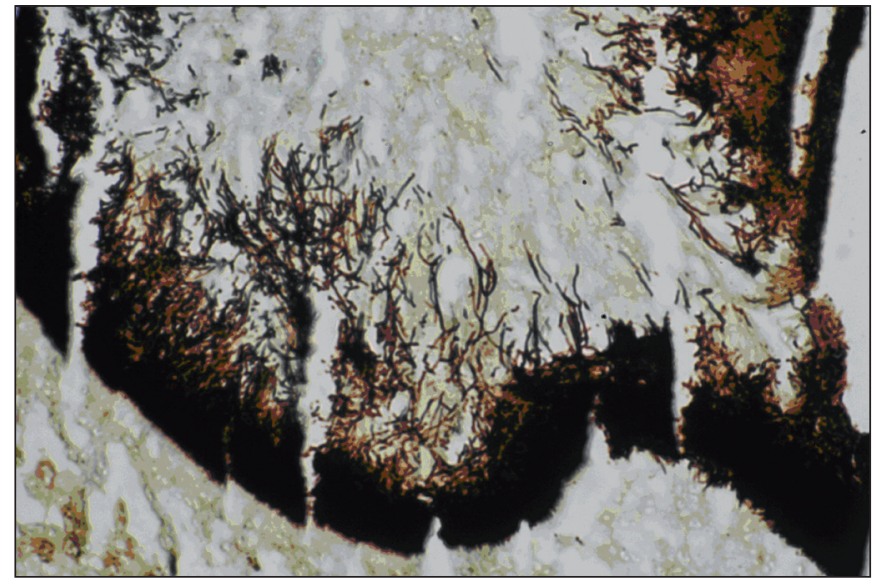

Figure 3: Filamentous bacilli stained GMS positive (GMS; x630). 
Radiologically, it is difficult to differentiate mandibular osteomyelitis from other neoplasias. Therefore, in such cases, bone lesion biopsy is important for determining the nature of the lesion.

Ultrasound is the primary preferred imaging technique that is used routinely for the neck region due to lack of side effects and rapid implementation. During ultrasound, fine needle aspiration cytology can be performed to achieve a pathological diagnosis. Computed tomography is more effective for identifying abscesses and surrounding anatomic structures; however, it may not fully reveal specific masses in soft tissue. Branchial cleft cyst, lymph node metastasis, inflammatory lymphadenopathy, and vascular tumors are the differential radiological diagnoses (7).

Clinical diagnosis is difficult due to non-specific findings. Differential diagnoses include many diseases, from tumors to chronic diseases (carcinoma, tuberculosis, and others). Cases are diagnosed based on the presence of bacteria with a positive culture and biopsy. Pathological diagnosis is simpler and less invasive using fine-needle aspiration cytology. Yellow sulfur granules are typically observed in the purulent material, which may be helpful in diagnosis. The drained material can be cultured; however, less than 50\% of the cultures are positive. Through the use of microscopy, colonies can be observed as filamentous bacilli with granular centers and a radial distribution (7). We diagnosed the mandibular mass as mandibular osteomyelitis caused by actinomycosis by observing positive filamentous bacilli with GMS, PAS, and Gram stain. Histochemical analysis of cervicofacial lesions can be used to determine the possibility of actinomycosis infection.

Bartkowski et al. reported 15 cases with actinomycotic osteomyelitis in the mandible. While nine of those cases were primary actinomycotic inflammation of the mandible, mandibular fracture or mandibular resection was observed in six of them. The authors reported that they obtained the diagnosis on the basis of bacteriological and histopathological tests (8). Additionally, in our case, oral hygiene was poor, and there were healed wounds and fistula scars on the skin. Our case differed from the other published cases because the lesion in our case had a mass image, and similar cases were not found in the literature. We used histopathology and histochemistry to achieve a diagnosis, and our outcome emphasized the importance of histopathology for diagnosing such lesions.

As a result, it is suggested that actinomycosis infections can mimic malignancy because in our case, the mass occurring in the mandible was clinically and radiologically evaluated primarily as a tumoral formation in the preoperative period. Ultrasound-guided fine-needle aspiration or biopsy will be very valuable for preoperative diagnosis of cervicofacial masses. Actinomycosis should be considered in patients with cervicofacial lesions because it may mimic malignancy. The removal of the infected bone, careful debridement of dental remains, and long-term intravenous and oral antibiotic therapy will be effective in the treatment of mandibular osteomyelitis caused by actinomyces.

\section{REFERENCES}

1. Figueiredo LM, Trindade SC, Sarmento VA, de Oliveira TF, Muniz WR, Valente RO. Actinomycotic osteomyelitis of the mandible: An unusual case. Oral Maxillofac Surg. 2013;17:299-302.

2. Kingdom TT, Tami TA. Actinomycosis of the nasal septum in a patient infected with the human immunodeficiency virus. Otolaryngol Head Neck Surg. 1994;111:130-3.

3. Baliga S, Shenoy S, Wilson G, Katara V. An unusual case of actinomycosis. Ear Nose Throat J. 2002;81:44-5.

4. Ozcan C, Talas D, Görür K, Aydin O, Yildiz A. Actinomycosis of the middle turbinate: An unusual cause of nasal obstruction. Eur Arch Otorhinolaryngol. 2005;262:412-5.

5. Vigliaroli E, Broglia S, Iacovazzi L, Maggiore C. Double pathological fracture of mandibula caused by actinomycotic osteomyelitis: A case report. Minerva Stomatol. 2010;59:507-17.

6. Acevedo F, Baudrand R, Letelier LM, Gaete P. Actinomycosis: A great pretender. Case reports of unusual presentations and a review of the literature. Int J Infect Dis. 2008;12:358-62.

7. Carinci F, Polito J, Pastore A. Pharyngeal actinomycosis: A case report. Gerodontology. 2007;24:121-3.

8. Bartkowski SB, Zapala J, Heczko P, Szuta M. Actinomycotic osteomyelitis of the mandible: review of 15 cases. J Craniomaxillofac Surg. 1998;26:63-7. 